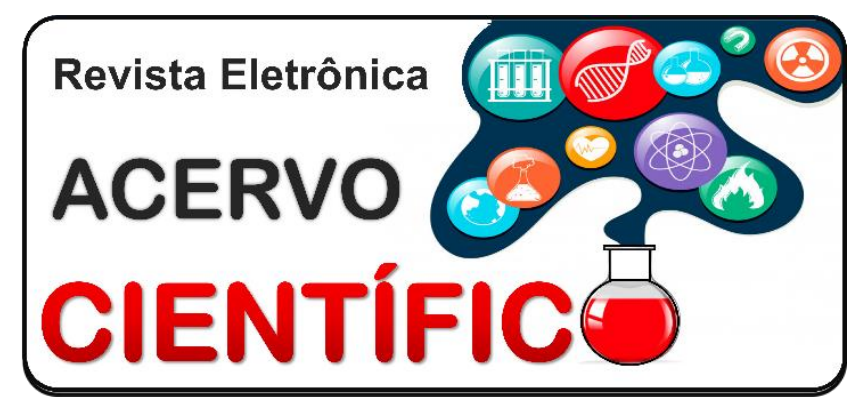

REVISÃO BIBLIOGRÁFICA

Recebido em: 7/2020

Aceito em: $8 / 2020$

Publicado em: 10/2020

\title{
Suplementação de colágeno e outras formas de tratamento no combate ao envelhecimento cutâneo
}

\author{
Collagen suplementation and other forms of treatments to combat skin aging \\ Suplementación de colágeno y otras formas de tratamiento para combatir el \\ envejecimiento de la piel
}

\begin{abstract}
Adriana Simões Ferreira ${ }^{1 *}$, Milena Ferreira Gandra ${ }^{1}$, Carolina de Almeida Freitas², Carolina Nunes Caro Varela ${ }^{3}$, Gabriela Cruz de Castro ${ }^{1}$, Karoline Barbosa Matias Silva ${ }^{1}$, Lizia de Carvalho Freitas Silva', Mariana Castro Soalheiro¹, Paula Quintão de Souza1, Taynna Tose Poncio¹.
\end{abstract}

\begin{abstract}
Resumo: Esse artigo buscou verificar, através de uma revisão de literatura, a eficácia da suplementação de colágeno dentre os vários métodos existentes no combate ao envelhecimento. O envelhecimento cutâneo é resultado da diminuição da função celular devido a fatores intrínsecos ou extrínsecos e suas principais consequências clínicas são caracterizadas por rugas, flacidez, hiperpigmentação, espessamento e aspereza. A perda do colágeno tem início na vida adulta e se intensifica a partir dos 40 anos. Fatores como radicais livres, dieta deficiente, alcoolismo, tabagismo e algumas doenças também contribuem para essa perda. São diversos os tratamentos para esse fim, sendo retratados nesse trabalho os benefícios das vitaminas C e E, a reposição hormonal, a micropuntura, o ultrassom microfocado ou macrofocado, o sculptra, o preenchimento com ácido hialurônico e a suplementação oral de colágeno na dieta, sendo este uma boa opção de escolha, uma vez que vem apresentando efeitos satisfatórios, permitindo uma boa aparência e aumentando a autoestima da população. É importante salientar, entretanto, que não se deve expandir esse método para todas as pessoas de maneira aleatória, de forma que cada paciente apresenta suas peculiaridades e uma indicação específica de terapêutica no combate ao envelhecimento.
\end{abstract}

Palavras-chave: Envelhecimento da pele, Terapêutica, Colágeno.

Abstract: This article aimed to verify, through a literature review, the efficacy of collagen supplementation among the various existing methods to fight aging. Skin aging is a result of decreased cellular function due to intrinsic or extrinsic factors and its main clinical causes, are characterized by wrinkles, flaccidity, hyperpigmentation, thickening and roughness. The loss of collagen initiates in adult life and intensifies around the age of 40 years old. Factors such as free radicals, poor diet, alcoholism, smoking and some diseases also contribute to this loss. There are several treatments for this purpose, being brought in this study the benefits of vitamins $\mathrm{C}$ and $\mathrm{E}$, hormonal replacement, micropuncture, microfocused ultrasound, or macrofocused, sculptptra, filling with hyaluronic acid and oral dietary supplementation, the last being a good

${ }^{1}$ Centro Universitário de Caratinga (UNEC), Caratinga - MG. *E-mail: adrianasimoes.f@hotmail.com

2 Universidade do Sul de Santa Catarina (UNISUL), Palhoça - SC.

${ }^{3}$ Universidade Nove de Julho (UNINOVE), Osasco - SP. 
choice since it brings satisfactory effects, allowing a good appearance and increasing population's selfesteem. It is important to emphasize, however, that this method should not be expanded to all people at random, so that each patient has its own peculiarities and a specific indication of therapy to fight skin aging.

Keywords: Skin aging, Therapeutics, Collagen.

Resumen: Este artículo buscó verificar, a través de una revisión de la literatura, la efectividad de la suplementación con colágeno entre los distintos métodos que existen para combatir el envejecimiento. El envejecimiento de la piel es el resultado de la disminución de la función celular debido a factores intrínsecos o extrínsecos y sus principales consecuencias clínicas se caracterizan por arrugas, flacidez, hiperpigmentación, engrosamiento y rugosidad. La pérdida de colágeno comienza en la edad adulta y se intensifica a partir de los 40 años. Factores como los radicales libres, la mala alimentación, el alcoholismo, el tabaquismo y algunas enfermedades también contribuyen a esta pérdida. Existen varios tratamientos para tal fin, en los que se retratan en este trabajo los beneficios de las vitaminas $C$ y E, reposición hormonal, micropunción, ultrasonido microfocalizado o macrofocalizado, sculptra, relleno con ácido hialurónico y colágeno oral en la dieta, siendo esta una buena opción de elección, ya que ha venido presentando efectos satisfactorios, permitiendo una buena apariencia y aumentando la autoestima de la población. Es importante señalar, sin embargo, que este método no debe extenderse a todas las personas al azar, por lo que cada paciente tiene sus propias peculiaridades y una indicación específica de terapia para combatir el envejecimiento.

Palabras clave: Envejecimiento de la piel, Terapéutica, Colágeno.

\section{INTRODUÇÃO}

O envelhecimento da pele é um fenômeno progressivo e degenerativo decorrente de alterações morfológicas e funcionais, cujo processo transcorre da redução de células epiteliais, importantes para manutenção da homeostasia do corpo. Tais alterações podem ocorrer sob a influência de fatores extrínsecos e intrínsecos (ADDOR FAS, 2015; BARBARA ECOS, et al., 2017).

O envelhecimento extrínseco é um processo relacionado a agressões externas que acumulam efeitos nocivos à pele. Vários são os fatores responsáveis por esse processo, dentre eles estão a poluição atmosférica, consumo de bebidas alcoólicas, tabagismo, hábitos alimentares, mas principalmente a exposição solar, condição conhecida como fotoenvelhecimento. A radiação solar em excesso promove a perda do suporte estrutural da pele, em decorrência da deficiência de colágeno e fibrilina na derme papilar. Outras condições relacionadas ao estilo de vida podem também contribuir para esse processo (BRAVO BSF, et al., 2014; ADDOR FAS, 2015; MESA-ARANGO AC, et al., 2017).

$\mathrm{Na}$ derme, devido ao envelhecimento intrínseco há uma redução da síntese de colágeno e outros componentes da matriz extracelular. Dessa forma, a cútis sofre perda de densidade e espessura e passa a ser caracterizada por flacidez e afinamento, dando indícios do envelhecimento cutâneo (ADDOR FAS, 2015; BARBARA ECOS, et al., 2017).

Trata-se de um processo fisiológico inevitável correlacionado à idade cronológica da pele. A degeneração é decorrente do declínio da função biológica e ação de espécies reativas de oxigênio derivados do metabolismo celular ao nível dérmico e epidérmico. Alguns autores afirmam que apenas $3 \%$ dos fatores que causam o envelhecimento cutâneo são intrínsecos, sendo a maior parte dos efeitos ocasionados por fatores de origem extrínseca (BRAVO BSF, et al., 2014; ADDOR FAS, 2015; ZHANG S e DUAN E, 2018).

O colágeno é a proteína mais importante produzida pelo organismo, sendo o fibrilar, o mais abundante, responsável em formar a estrutura da pele, sua morfologia e propriedades mecânicas como força tênsil e resistência (LEÓN-LÓPEZ A, et al., 2019). Sabe-se que a perda de colágeno pode ter início a partir do intervalo de idade entre 18 e 29 anos. Após os 40 anos a perda se acentua e pode chegar a $1 \%$ ao ano, e 
por volta dos 80 anos a produção de colágeno já se reduziu em $75 \%$ quando comparada a um adulto jovem (LEÓN-LÓPEZ A, et al., 2019).

Os tratamentos para essa perda são diversos, podendo ser eles mais ou menos invasivos, tópicos ou orais. Um deles é o uso de suplementos orais contendo colágeno. Eles geralmente contêm colágeno hidrolisado ou peptídeos de colágeno, sendo o último de mais fácil absorção. O objetivo é melhorar a estrutura da derme, assim como a espessura, elasticidade e hidratação (BRAVO BSF, et al., 2014; ADDOR FAS, 2015). A autopercepção sobre a saúde possui impacto sobre o processo de envelhecimento, pois proporciona um determinado estilo de vida que leva o indivíduo a tomar ações de autoproteção para um viver saudável (MARI FR, et al., 2016).

As consequências do envelhecimento são principalmente psicológicas pois estão relacionadas com a autoestima. Assim, todos desejam uma aparência jovem, uma vez que existe influência no comportamento social. Portanto, muitas pessoas, especialmente as mulheres, estão sempre em busca de tratamentos que previnam e/ou revertam o envelhecimento cutâneo, sendo assim uma importante área de pesquisa (ZHANG S e DUAN E, 2018).

O colágeno tem sido consumido como suplemento na dieta como um recurso para a saúde da pele (KIM D, et al., 2018). Por proporcionar supostos benefícios na redução de rugas, rejuvenescimento, reversão do envelhecimento e da queda da pele, têm sido amplamente comercializado e se tornaram um tratamento popular e moderno, (JHAWAR N, et al., 2020).

Sendo assim, o objetivo do presente trabalho é verificar, por meio de uma revisão de literatura, se esse tipo de tratamento é uma boa opção de escolha dentre os vários métodos existentes no combate ao envelhecimento cutâneo por perda de colágeno.

\section{REVISÃO BIBLIOGRÁFICA}

O envelhecimento cutâneo é resultado da diminuição da função celular devido a fatores intrínsecos ou extrínsecos, esses fatores atuam simultaneamente induzindo mudanças na pele (MESA-ARANGO AC, 2017). O tecido conjuntivo é perdido, isso leva diminuição e perda da elasticidade e tom de pele. As alterações que ocorrem podem ser classificadas em orgânicas e não orgânicas (BARATI M, et al., 2020).

Das alterações não orgânicas, destaca-se a alteração psicológica, pois está diretamente relacionada à autoestima (ZHANG S e DUAN E, 2018). Essa autopercepção sobre a saúde possui impacto sobre o processo de envelhecimento, pois proporciona um determinado estilo de vida que leva o indivíduo a tomar ações de autoproteção para um viver saudável. Grupos de mulheres, pessoas de maior idade com baixa escolaridade e negros são mais propensos a obter uma percepção negativa sobre a própria saúde devido a fatores sociais, culturais, psicológicos e ambientais. Com o decorrer do envelhecimento os grupos sociais passam a apresentar mais limitações físicas e acendem uma preocupação maior com a estética como sinais de pele e cabelos brancos. A preocupação com a longevidade está relacionada com a melhoria nas condições de vida e a responsabilização com o autocuidado através de uma boa alimentação, prática de atividade física, cuidados consigo próprio e cuidados médicos (MARI FR, et al., 2016).

Das alterações orgânicas, observam-se mudanças macroscópicas e microscópicas na pele, sendo a primeira representada pelas queimaduras, eritema, hiperpigmentação, telangectasias, pele seca, rugas profundas, perda da tonalidade natural ou câncer de pele e, a segunda, caracterizada por manifestações nas células cutâneas, como alterações fenotípicas, estruturais e funcionais nos componentes da matriz extracelular, incluindo colágenos e elastina (ZHANG S e DUAN E, 2018).

A molécula de colágeno é constituída por uma sequência repetida de três aminoácidos ( $A A)$ (Gly-X-Y), sendo Gly a glicina, $X$ na maioria das vezes é a prolina e $Y$ hidroxiprolina ou hidroxilisina. Esses $A A$ não essenciais se organizam basicamente em $30 \%$ de glicina, $12 \%$ de prolina, $11 \%$ de alanina, $10 \%$ de hidroxiprolina e $1 \%$ de hidroxilisina para formação do colágeno, que é uma proteína com qualidade inferior do ponto de vista nutricional, devido a predominância mínima ou ausente dos AA essenciais triptofano, metionina, cistina e tirosina, que se dividem formando os $36 \%$ restantes. Ainda que haja esse pequeno valor 
nutricional, a diversidade dos aminoácidos não essenciais é importante para síntese de colágeno na matriz extracelular de alguns tecidos e nas cartilagens (PORFÍRIO E e FANARO GB, 2016).

A perda do colágeno no corpo se dá entre 18 e 29 anos, sendo cerca de 1\% ao ano após 40 anos e $75 \%$ no geral depois dos 80 anos, em comparação aos adultos jovens. Além da idade, fatores como radicais livres no organismo, dieta deficiente, alcoolismo, tabagismo e algumas doenças, também contribuem para essa perda (LEÓN-LÓPEZ A, et al., 2019). A menopausa também é um fator que contribui para as alterações degenerativas do colágeno e espessura dérmica. Gradualmente, a diminuição do colágeno, alcança o pico mais alto nos primeiros cinco anos (até 30\%) e, em seguida, ocorre 1-2 \% ao ano (ADDOR FAS, 2015).

O avanço biotecnológico, associado à significativa melhoria na qualidade de vida tem permitido que as pessoas alcancem estágios de desenvolvimento cada vez mais avançados no ciclo de vida (AGUIAR A, 2018). Atualmente, a indústria cosmética passa por um crescimento dinâmico, devido à busca cada vez maior por uma aparência jovem e saudável, que está diretamente relacionada com a melhora da qualidade de vida dos consumidores (CAMPOS PMBGM, et al., 2016).

De acordo com os estudos de Mari FR, et al. (2016), os adultos apresentaram maior preocupação com a estética e o efeito que pode causar diretamente no convívio social, e também referiram ter medo do processo de envelhecimento, como sinais de pele e cabelos brancos. Já os idosos, se atentam mais as limitações físicas. Contudo ambos mostram preocupação com esse processo.

São diversos os tratamentos para a perda de colágeno, incluindo procedimentos mais ou menos invasivos, orais ou tópicos. Dentre esses métodos podemos citar a utilização de antioxidantes como o ácido ascórbico (vitamina C) e tocoferol (vitamina E), a terapia de reposição hormonal, a micropuntura, o ultrassom microfocado ou macrofocado, o sculptra, o preenchimento com ácido hialurônico, além da suplementação oral de colágeno (BRAVO BSF, et al., 2014).

Com o envelhecimento, espécies reativas de oxigênio e radicais livres são acumulados na pele, danificando suas células. Esses danos, induzem a formação de aldeídos, hidroxilação, reticulação e adição de radicais. Os produtos de antienvelhecimento tópico, em sua maioria, funcionam diminuindo a degradação dos elementos que estruturam a pele, como a elastina, colágeno e ácido hialurônico, podendo também estimular a síntese desses compostos. Os anti-oxidantes que incluem genisteína, vitamina $\mathrm{C}$ e vitamina $\mathrm{E}$, podem evitar a inflamação da pele e diminuir os danos existentes. A utilização da vitamina $E$ tem sido apoiada, mas ainda precisam ser realizados mais estudos. Já a vitamina $C$ está em uso no tratamento da pele de idosos, porém as preparações incluem ésteres solúveis que penetram a pele facilmente, ao invés do ácido ascórbico, que é a forma ativa da vitamina $C$, e está envolvida na conversão do procolágeno em colágeno (CALLEJA-AGIUS J, 2013).

$\mathrm{Na}$ menopausa, ocorre a queda de estrogênio no corpo e, com isso, uma redução do colágeno, causando várias alterações na pele, como pele seca, rugas, afinamento e diminuição da elasticidade. Para minimizar essas alterações pode-se optar pela terapia de reposição hormonal (TRH), que leva ao aumento da espessura da pele, aumento do conteúdo de colágeno e ácido hialurônico da derme. Dessa forma, as mulheres que fazem terapias hormonais possuem a pele com aparência mais saudável do que a pele de mulheres da mesma idade que não fazem TRH (CALLEJA-AGIUS J, 2013).

Apesar de inicialmente ter observado melhoras estéticas, com o passar do tempo os estudos de controle mostraram que a TRH aumenta o risco de desenvolver câncer de mama e a prescrição para esse tratamento ficou mais restrito e utilizado apenas no tratamento de osteoporose. Atualmente, em vez do $\mathrm{TRH}$, o tratamento com estrógeno é preferido para tratar a pele envelhecida, já que existe uma forte correlação entre a deficiência de estrógeno e a perda de colágeno, sendo a terapia tópica com estrogênio segura quando prescrita por dermatologista. Essa terapia tem melhor resultado quando a administração da dose e o tempo de tratamento, em torno de 2 anos, é feito de forma correta sendo $1,8 \mathrm{~g}$ por dia durante 8 semanas. Caso a dose seja muito alta ou muito baixa ocorre um aumento no colágeno da pele, porém, em menor grau quando comparada a dosagem apropriada (CALLEJA-AGIUS J, 2013). 
Uma outra técnica para o tratamento de envelhecimento pela perda de colágeno existente, é a micropuntura, em que se utiliza um aparelho de dermógrafo com uma agulha estéril que realiza sucessivas perfurações na pele. Esse tratamento é indicado para minimizar linhas de expressão, rugas faciais, cicatrizes de acne e estrias. Entretanto, não deve ser realizado em gestantes, lactantes, pessoas portadoras de diabetes, pacientes oncológicos, pessoas que fazem uso de medicamentos vasodilatadores, antiinflamatórios e anticoagulantes e que possuem alergia a metais. O aumento da produção de colágeno e elastina na região de trauma exercido pela penetração da agulha, faz com que haja redução da ruga. $O$ processo inflamatório após a aplicação da micropuntura, pode ser dividido em três fases: a primeira é a fase inflamatória, que ocorre logo após a lesão, em seguida vem a fase proliferativa, onde tem o fechamento da lesão e a terceira é a fase de remodelagem onde há tentativa de recuperação tecidual, e a matriz celular é organizada. Ainda nessa terceira fase a reposição do colágeno de forma alinhada é evidente, sendo o colágeno inicial substituído por um mais espesso e organizado, gerando uma boa cicatrização e dando aumento da força tênsil na ferida. A aplicação dessa técnica deve ter intervalo mínimo de 7 dias e máximo de 14 dias, para que o processo de inflamação aguda seja absorvido pelo organismo (BARBARA ECOS, et al., 2017).

Atualmente, têm sido utilizado equipamentos emissores de ultrassom, para fornecer energia térmica no tecido conjuntivo dérmico profundo adicionalmente à derme superficial, o que induz a uma remodelação do colágeno mais completa do que os métodos anteriores (BANI D, et al., 2014). A técnica de Ultrassom Micro ou macrofocado emite uma energia em forma de cone capaz de atingir um ponto focal, fazendo com que haja a formação de um novo colágeno seguido de tensão do tegumento. Essa metodologia de tratamento, se executada de forma correta, pode levar a uma redução significativa e durável das rugas cutâneas, de acordo com evidências clínicas (BAZZO KDL, 2019). Além disso, os efeitos colaterais do ultrassom são desprezíveis, consistindo principalmente em eritemas transitórios, edemas e dor moderada. Desse modo, possui uma excelente adesão por partes dos pacientes (BANI D, et al., 2014).

Existem também outros tipos de tratamento que são bioestimuladores de colágeno, como o Sculptra que são injeções contendo o ácido poli-I-láctico, que ao ser injetado na pele, produz resposta inflamatória local subclínica e, à medida que esse ácido é metabolizado, ocorre a deposição aumentada de colágeno produzida pelo fibroblasto e, consequentemente, o aumento da espessura dérmica. Também são realizadas técnicas minimamente invasivas com preenchedores, volumizadores e estimuladores (FILHO CASM, et al., 2013). O ácido hialurônico é um tipo de preenchedor, que possui propriedades elásticas que oferecem resistência à compressão da pele, protege de danos mecânicos existentes e permite que fibras colágenas se movam facilmente. Sendo assim, é uma peça essencial no tratamento do rejuvenescimento cutâneo (BERNARDES IN, et al., 2018).

O uso tópico e oral do colágeno é amplamente popularizado e afirma-se possuir propriedades antienvelhecimento nos últimos anos (WICH S, 2020). No mundo da saúde da pele, os suplementos de colágeno oral tornaram-se um tratamento popular e moderno. Por proporcionar supostos benefícios na redução de rugas, rejuvenescimento, reversão do envelhecimento e da queda da pele, tem sido amplamente comercializado (JHAWAR N, et al., 2020).

A ingestão do colágeno não se difere das demais proteínas, uma vez que não é absorvido como colágeno, senão que sob a forma de AA. A digestão protéica é realizada $80 \%$ no duodeno e jejuno pela ação do suco pancreático, enquanto $10-20 \%$ ocorre no estômago com a ajuda do ácido clorídrico e da pepsina. A hidrólise luminal de proteínas e polipeptídeos em AA livres e pequenos peptídeos pela ação da enteropeptidase, que devido ao $\mathrm{pH}$ neutro, ativa o tripsinogênio e a tripsina, que promove a ativação das outras proptidases do suco pancreático, toda essa reação de hidrólise luminal ocorre também no intestino delgado. Os AA são destinados a inúmeras funções, inclusive para a síntese do próprio colágeno. A partir do colágeno tipo 1, que é abundante no tecido conjuntivo, são obtidos o colágeno parcialmente hidrolisado (gelatina) e o colágeno hidrolisado, sendo a diferença entre eles é que o colágeno hidrolisado dissolve em água ou salmora, tornando fácil a digestão e absorção, assim como a produção de colágeno pelo organismo a partir de AA livres. Além disso, o colágeno hidrolisado possui em sua composição glicina e prolina, que são essenciais para a estabilidade e regeneração das cartilagens (PORFÍRIO E e FANARO GB, 2016). 
O colágeno hidrolisado tem sido muito estudado pelo seu potencial benéfico biológico sobre 0 metabolismo da pele (ZANGUE V, et al., 2016). Na derme existem duas formas diferentes de atuação do colágeno hidrolisado, inicialmente os aminoácidos livres fornecem blocos de construção para a formação de fibras de colágeno e elastina. Em seguida, os oligopeptídeos de colágeno atuam como ligantes, ligando-se a receptores na membrana dos fibroblastos e estimulando a produção de novo colágeno, elastina e ácido hialurônico (LEÓN-LÓPEZ A, et al., 2019).

Considerando que o colágeno é um nutriente estrutural e desnutrido naturalmente, uma dieta rigorosa ou uma síndrome de má absorção obviamente se beneficiarão da suplementação de proteínas (ADDOR FAS, 2015). Esse suprimento atinge as camadas mais profundas da pele, melhorando sua aparência e fisiologia, além de aumentar a hidratação, elasticidade, firmeza, redução e rejuvenescimento da pele (LEÓN-LÓPEZ A, et al., 2019). O aparecimento de uma nova geração na suplementação do colágeno com peptídeos específicos é capaz de aumentar a expressão de determinadas moléculas ligadas à síntese colagênica e associações com outras substâncias, como vitaminas e fitoextratos, que atuariam de forma sinérgica, potencializando o efeito de antienvelhecimento (ADDOR FAS, 2015). Sendo assim, é um produto seguro, industrial de venda livre e retém a autorização da Agência Nacional de Vigilância Sanitária (ANVISA) para a comercialização ao público coletivo (ZAGUE V, 2016; JACKIX EA, 2008).

\section{CONSIDERAÇÕES FINAIS}

Por meio da análise bibliográfica deste estudo pode-se concluir que dentre os diversos tipos de tratamentos para o envelhecimento da pele por perda de colágeno, o uso do suplemento oral na dieta apresenta-se como uma boa opção de escolha, uma vez que vem apresentando efeitos satisfatórios na pele, permitindo uma boa aparência e aumentando a autoestima da população. Além de ser um procedimento não invasivo, de fácil administração, o que aumenta as chances de sucesso na adesão ao tratamento pelo paciente. É importante salientar, entretanto, que não se deve expandir esse método para todas as pessoas de maneira aleatória, de forma que cada paciente apresenta suas peculiaridades e uma indicação específica de terapêutica medicamentosa no combate ao envelhecimento. Por esse motivo, reforça-se da necessidade de investimentos de estudos científicos nessa área, visando uma melhora no âmbito clínico, redução dos custos e ampliação de seu uso.

\section{REFERÊNCIAS}

1. ADDOR FAS. Influence of a nutritionl supplement containing collagen peptides on the properties of the dermis. Surgical and Cosmetic Dermatology, 2015; 7(2): 116-121.

2. AGUIAR A, et al. Envelhecimento e Prática de Rejuvenescimento: Estudo de Representações Sociais. Psicol. cienc. prof., Brasília, 2018; 38 (3): 494-506.

3. BANI D, et al. Efeitos do tratamento de ultrassom de alta freqüência sobre os tecidos da pele humana. Surg. Cosmet. Dermatol., 2014; 6(2): 13846.

4. BARATI M, et al. Suplementação de colágeno para a saúde da pele: Uma revisão sistemática mecanicista, 2020.

5. BARBARA ECOS, et al. Uso da micropuntura no tratamento de rugas. Fisioterapia Brasileira, 2017; $18(4)$ : 481 -488.

6. BAZZO KDL. Utilização do ultrassom microfocado no tratamento dos sinais de idade. Biblioteca digital de tcc- Curso de Fisioterapia, Faculdade União das Americas, Paraná, 2019.

7. BERNARDES IN, et al. Preenchimento com ácido hialurônico - revisão de literatura. Revista Saúde em Foco. 2018; 10: 603-612.

8. BRAVO BSF, et al. Oral isotretinoin in photoaging: objective histological evidence of efficacy and durability. Anais Brasileiros de Dermatologia, 2015; 90 (4): 478-486.

9. CALLEJA-AGIUS J, et al. Tecido conjuntivo de pele e envelhecimento. Pract. Res. Clin. Obstet. Gynaecol., 2013; 27(5): 727-740.

10. CAMPOS PMBGM, et al. Suplementação Oral de um Nutricosmético à base de peptídeos de colágeno sobre a elasticidade e a ecogenicidade dérmica: estudo clínico duplo-cego, placebo-controlado. Clinical Pharmacology e Biopharmaceutics, 2015; 4(3).

11. FILHO CASM, et al. Ácido PoliLLáctico: um agente bioestimulador. Surg. Cosmet. Dermatol., 2013; 5(4): 34550. 
12. JACKIX EA. Efeito da suplementação alimentar com hidrolisado de colágeno nos mercadores bioquímicos e nas características composicionais, biomecânicos e histológica óssea de ratas osteopênicas. Dissertação (Mestrado) Curso de Engenharia de Alimentos, Universidade Estadual de Campinas, Campinas, 2008.

13. JHAWAR N, et al. Suplementação oral de colágeno para envelhecimento da pele: Uma moda ou o futuro? J. Cosmet. Dermatol., 2020; 19(4): 910-912.

14. KIM D, et al. Oral intake of low-molecular-wight collagen peptide improves hydration, elasticity and wrinkling in human skin: A randomized, double-blind, placebo-controlled study. Nutrients, 2018; 10 (7): 826.

15. LEÓN-LÓPEZ A, et al. Hydrolyzed Collagen—Sources and Applications. Molecules, 2019; 24(22): 4031.

16. LIN P, et al. Oral Collagen Drink for Antiaging: Antioxidation, Facilitation of the Increase of Collagen Synthesis, and Improvement of Protein Folding and DNA Repair in Human Skin Fibroblasts. Oxid. Med. Cell. Longev., 2020.

17. MARI FR, et al. O processo de envelhecimento e a saúde: o que pensam as pessoas de meia-idade sobre o tema. Revista brasileira geriatria gerontologia, Rio de Janeiro, 2016; 19 (1): 35-44.

18. MESA-ARANGO AC, et al. Mechanisms of skin aging. Latreia, 2017; 30 (2): 160-170.

19. PORFIRIO E, FANARO GB. Suplementação com colágeno como terapia complementar na prevenção e tratamento de osteoporose e osteoartrite: uma revisão sistemática. Revista Brasileira de geriatria e gerontologia, Rio de Janeiro, 2016; 19 (1): 153-164.

20. WICH S, PRAVIT A. Four-weeks daily intake of oral collagen hydrolysate results in improved skin elasticity, especially in sun-exposed areas: a randomized, double-blind, placebo-controlled trial. Journal of Dermatological Treatment, 2020.

21. ZAGUE V. Influência da suplementação com colágeno hidrolisado no metabolismo da matriz extracelular e proliferação de fibroblastos dérmicos humanos derivados de áreas fotoprotegida e fotoexposta, cultivados em monocamada e equivalente dérmico. Tese (Doutorado) - Curso de Biologia Celular, Universidade de São Paulo, São Paulo, 2015.

22. ZANGUE V, MACHADO-SANTELLI GM. Bases Científicas dos Efeitos da Suplementação Oral com Colágeno Hidrolisado na Pele. Revista Brasileira de Nutrição Funcional, 2016.

23. ZHANG S, DUAN E. Fighting against skin aging: The way from bench to bedside. Cell Transplantation, $2018 ; 27$ (5): 729-738. 